\title{
Bio-inspired structurally colored materials
}

Ming Xiao

Harvard University, United States

Structural colors are produced by coherent scattering from nanostructures with correlations at a length scale comparable to visible wavelengths. They are resistant to photo bleaching, chemical washing, and feasible to be made of environmentally friendly materials, in contrast to pigmentary colors. Application of structural colors requires optimized optical properties, multiple functionalities, and scalable fabrication. One approach to solve these issues is to seek inspirations from nature, where there are many amazing structurally colored systems, like butterfly wings, bird feathers, and beetle scales.

In this talk, we will present two stories on how we design and engineer structurally colored materials on demand by mimicking both structure and function of biological photonic systems. First, inspired by nanostructures in bird feathers and guided by optical simulations, we design core-shell melanin nanoparticles to enhance brightness in structural colors. ${ }^{1}$ In addition, the unique broadband absorption of melanin leads to high saturation. ${ }^{2}$ We then assemble these core-shell nanoparticles to structurally colored supraballs using a scalable one-pot reverse emulsion process. The photonic supraballs can be easily used as colorful inks for coating or printing. ${ }^{3}$ Second, inspired by fast color changing in cephalopods and angleindependent colors in blue cotinga, we develop a wide-angle color-changing platform by integrating photonic glass to a dielectric elastomer. It can not only alter quickly and reversibly change colors with the electric field, but also undergo shape changing due to out-of-plane deformation (i.e. buckling or wrinkling). The use of photonic glass allows homogenous color changes under out-of-plane deformation and this is not possible with photonic crystal because out-of-plane deformation involves a gradient in surface normal, and thus leads to many different colors being reflected. To conclude, we demonstrate that the biomimetic approach provides a unique prospective to address current scientific and technology challenges.

\section{References}

(1) Xiao, M.; Hu, Z.; Wang, Z.; Li, Y.; Tormo, A. D.; Thomas, N. L.; Wang, B.; Gianneschi, N. C.; Shawkey, M. D.; Dhinojwala, A. Bioinspired Bright Noniridescent Photonic Melanin Supraballs. Sci. Adv. 2017, 3 (9), e1701151. https://doi.org/10.1126/sciadv.1701151.

(2) Xiao, M.; Shawkey, M. D.; Dhinojwala, A. Bioinspired Melanin-Based Optically Active Materials. Adv. Opt. Mater. 2020, 8 (19), 2000932. https://doi.org/10.1002/adom.202000932.

(3) Hu, Z.; Sun, H.; Thompson, M. P.; Xiao, M.; Allen, M. C.; Zhou, X.; Ni, Q. Z.; Wang, Z.; Li, W.; Burkart, M. D.; Deheyn, D. D.; Dhinojwala, A.; Shawkey, M. D.; Gianneschi, N. C. Structurally Colored Inks from Synthetic Melanin-Based Crosslinked Supraparticles. ACS Mater. Lett. 2021, 3 (1), 50-55. https://doi.org/10.1021/acsmaterialslett.0c00432. 